\section{Maximum control}

These days, the lactose system of Escherichia coli is regarded as the classic example of an operon. It is used by every modern molecular biology or genetics textbook worth its salt to illustrate the basic principles of gene transcription. The regulation of the structural genes lacZ, lacY and $l a c A$ through binding of the lacI-encoded repressor to the operator site $(\mathrm{lacO})$ was famously uncovered by Jacob and Monod at the beginning of the 1960s (see Milestone 2). However, it would take the rest of the decade, and some complex bacterial genetics, before their fellow scientists uncovered the key cis-regulatory element of transcription the promoter.

Even when they are fully induced, different enzymes are present in bacterial cells in widely varying amounts. So what controls the maximum rate of enzyme production? Scaife and Beckwith subscribed to the idea that gene expression could be controlled by a specific chromosomal site that is able to limit the rate of initiation of either transcription or translation. They therefore tried to identify such a site by searching for mutations that would decrease or abolish the potential of the lac operon to be expressed. Several mutations that resulted in reduced levels of all three lac structural genes were picked up in a mutagenesis screen. The researchers showed that these mutations were cis-dominant by confirming that their effects were not relieved by the introduction of a second lac region into the cell. Furthermore, using gene-repression experiments and detailed genetic analysis, they showed that these mutations were separate from the repressor-operator system, and mapped outside lacI and probably also outside $\mathrm{lacO}$.

By 1968, Ippen and colleagues had built on these initial results using a series of mutants in which increasing amounts of the lac operon and its upstream region had been deleted in a stepwise fashion. Recombination analysis showed that the mutations mapped to just before the beginning of the lac operon. The authors designated the site of the mutations as the 'promoter' (reintroducing a term that had been used previously, with a different meaning, by Jacob, Ullman and Monod). So did the promoter control transcription or translation?

Ippen and co-workers suggested that it is transcription that begins at the promoter.

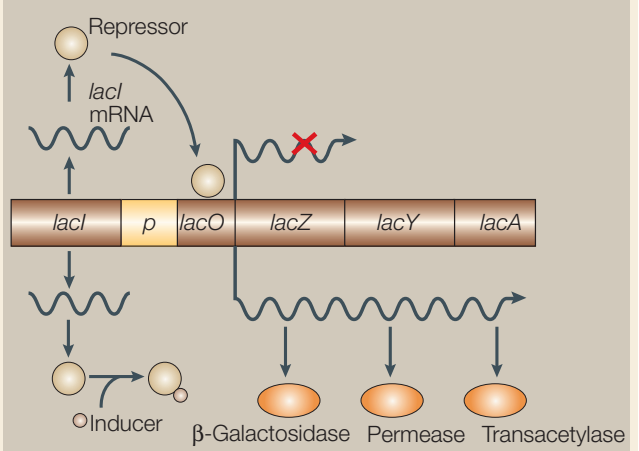

The lacl-encoded repressor binds to the lacO operato sequence and prevents transcription of the lac structural genes (top). Binding of an inducer prevents the repressor from binding $\mathrm{lacO}$ and the structural genes are transcribed (bottom). Studies using the lac operon identified the promoter $(p)$ as a cis controlling element for gene promoter $(p)$ as a cis controli
transcription in the 1960s.

Moreover, as there was no evidence that the lac $\mathrm{O}$ region is translated, they proposed that translation begins just downstream of lacO, in a region that Jacob, Ullman and Monod had previously suggested was important for gene expression.

The function of the promoter was elegantly confirmed by Eron and Block in 1971 using an in vitro transcription system. They showed that mutations in the lac promoter altered the levels of transcription initiation, and confirmed the functional significance of transcription initiation in vitro by showing that it required the auxiliary sigma factor (see Milestone 6), was negatively regulated by the lac repressor, and was positively regulated by cyclic AMP (cAMP) and cAMP-binding protein (see Milestone 4). Furthermore, confirming extensive genetic work from the Beckwith group, they showed that the lac promoter had two parts: one that mediated transcription initiation and one that mediated positive regulation. So, by the start of the 1970s, the concept of the promoter, and its potential for complexity of both regulation and structure, had emerged.

Lesley Cunliffe, Locum Associate Editor Nature Reviews Molecular Cell Biology

References and links

ORIGINAL RESEARCH PAPERS Scaife, J. \& Beckwith, J. R. Mutational alteration of the maximal level of lac operon expression. Cold Spring Harbor Symp. Quant. Biol. 31 403-408 (1966) | Ippen, K., Miller, J. H., Scaife, J. \& Beckwith, J. New controlling element in the lac operon of E. coli. Nature 217, 825-827 (1968) | Eron, L. \& Block, R. Mechanism of initiation and repression of in vitro transcription of the lac operon of Escherichia coli. Proc. Natl Acad. Sci. USA 68, 1828-1832 (1971)

FURTHER READING Beckwith, J., Grodzicker, T. \& Arditti, R. Evidence for two sites in the lac promoter region. J. Mol. Biol. 69, 155-160 (1972) | Gilbert, W., Maizels, N. \& Maxam, A. Sequences of controlling regions of the lactose operon. Cold Spring Harbor Symp. Quant. Biol. 38, 845-855 (1974) | Dickson, R., Abelson, J., Barnes, W. \& Reznikoff, W. Genetic regulation: the lac control region. Science 187, 17-35 (1975)

\section{Sigma, from the start}

It's easy to forget that somewhere between the now high-tech fields of genomics and transcriptomics once lay the fundamental problem of how DNA is transcribed. Perhaps most important was the question of how RNA polymerase is guided to the right promoters and prevented from binding unproductively to random sequences. This question was answered almost 40 years ago for bacteria with the finding that sigma factors lead RNA polymerase to its target sequences, thereby kick-starting a flurry of biochemical studies of transcription.

In 1969, knowledge about bacterial transcription was based largely on in vitro assays using purified RNA polymerase and a (typically bacteriophage) template DNA. Such experiments had shown that the polymerase was specific about the sequences it transcribed in vivo, but was less choosy in vitro - in fact, its specificity in vitro varied depending on the template used and how the polymerase was purified.

The solution to this confusing state of affairs depended on having a reliable means

\section{MILESTONE}

\section{And then there were three}

In bacteria, a single RNA polymerase core enzyme is known to be responsible for synthesizing all RNAs, including messenger RNAs (mRNAs), ribosomal RNAs (rRNAs) and transfer RNAs (tRNAs). However, until the early 1970s, it was unclear whether the same was true for eukaryotic cells.

At the time, several lines of evidence implied that a distinct polymerase transcribed rRNAs. The $(\mathrm{G}+\mathrm{C})$-rich base composition of rRNAs was distinct from other RNA classes, their genes were heavily redundant and localized in the nucleolus, and rRNA synthesis was regulated independently from the other RNA types.

In late 1969, Roeder and Rutter reported the discovery of three chromatographically separable forms of eukaryotic RNA polymerase from sea urchin embryos (I, II and III) and two species from rat liver (I and II). Further 
of separating an active RNA polymerase into its components and ascribing a function to each of them. Richard Burgess and Andrew Travers at Harvard, and John Dunn and Ekkehard Bautz at Rutgers, did just that: they purified Escherichia coli RNA polymerase on a phosphocellulose column and asked which combination of fractions, and then which factor in particular, conferred transcriptional activity to the core enzyme on T4 phage DNA.

This was the landmark paper that identified that the selective fraction was a protein - a result that was achieved largely thanks to the ability of a new biochemical technology, SDS-PAGE, to separate the protein components by size. Yet how this protein, which they called sigma, worked could only be speculated about: did it simply increase the affinity of the enzyme for any DNA, or was it more specific? And how and where did it associate with the enzyme?

In a second paper, Travers and Burgess showed that sigma increased the number of RNA chains that were initiated by the polymerase. In particular, as Dunn and Bautz pointed out in a third study, sigma functions just before the first RNA phosphodiester bond is formed. In experiments that were to alter how we view regulatory protein-protein interactions,

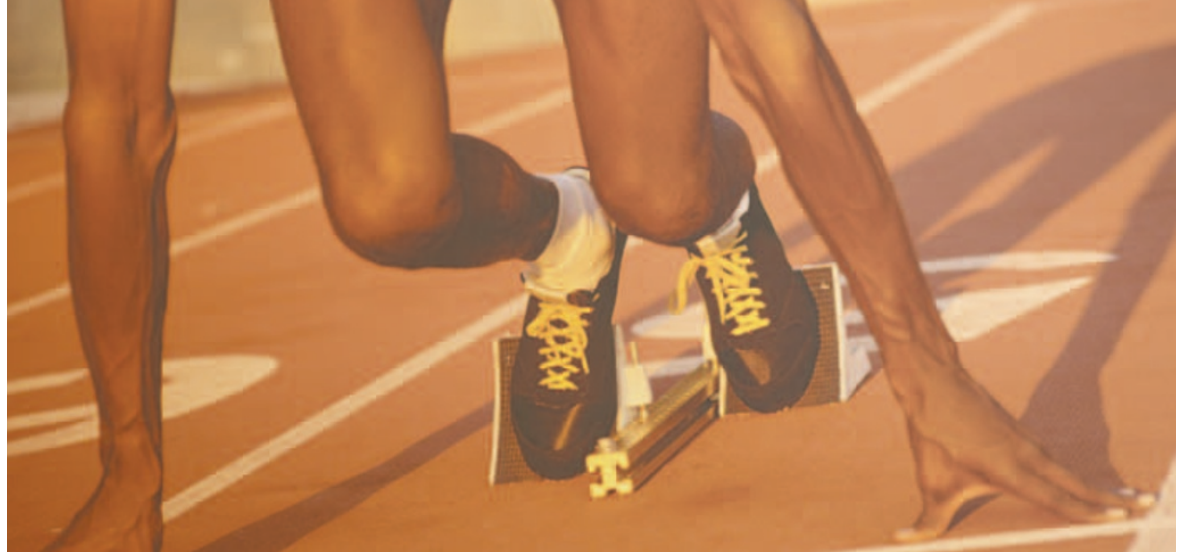

Travers and Burgess then showed that sigma is released by the polymerase after initiation and then reassociates with another polymerase enzyme in a cyclical fashion. These conclusions were reinforced by work in a different system by Joseph Krakow and colleagues, who reported that a protein component equivalent to sigma is released from the polymerase of the bacterium Azotobacter vinelandii.

These studies - along with work from the groups of Michael Chamberlin and Wolfram Zillig, who reported further evidence for sigma initiation factors at about the same time - spurred decades of study into promoter recognition and transcription initiation. Although a different mechanism operates in eukaryotes (see Milestone 12), the cyclical model of transcription has been amply validated and expanded in recent years, both as a general strategy for how auxiliary factors operate in transcription and through the identification of a family of prokaryotic sigma factors that determine the different promoter specificities of core polymerase enzymes.

Tanita Casci, Senior Editor, Nature Reviews Genetics

\section{References and links}

ORIGINAL RESEARCh PAPERS Burgess, R., Travers, A. A. Dunn, J. J. \& Bautz, E. K. Factor stimulating transcription by RNA polymerase. Nature 221, 43-46 (1969) | Travers, A. \& Burgess, R. Cyclic re-use of the RNA polymerase sigma factor. Nature 222, 537-540 (1969) | Dunn, J. \& Bautz, E. DNA-dependent RNA polymerase from E. coli: studies on the role of sigma in chain initiation. Biochem. Biophys. Res. Commun. 36, 925-930 (1969)|Krakow, J. S., Daley, K. \& Karstadt, M. Azotobacter vinelandii RNA polymerase, VII. Enzyme transitions during unprimed $\mathrm{r}[\mathrm{l}-\mathrm{C}]$ synthesis. Proc. Natl Acad. Sci. USA 62, 432-437 (1969)

FURTHER READing Berg, D., Barrett, K., Hinkle, D. McGrath, J. \& Chamberlin, M. A subunit of RNA polymerase involved in chain initiation. Fed. Proc. 28, 659 (1969) | Sethi, V. S. Zillig, W. \& Bauer, H. Dissociation and reconstitution of active DNA-dependent RNA-polymerase from E. coli. FEBS Lett. 8 , 236-239 (1970) characterization of the polymerases showed differing salt requirements for maximal activity, and indicated that sea urchin polymerase I and II were similar to their respective enzymes in rat. The authors proposed that polymerase I and II localized primarily in the nucleolus and the nucleoplasm, respectively. Furthermore, they proposed that increases in polymerase I levels were responsible for observed increases in rRNA levels during gastrulation. However, it was still not known whether the three forms of polymerase were the products of three distinct genes or simply the result of the differential regulation of a single gene product.

Work published by the Chambon laboratory a few months later began to clarify things. They examined the ability of the toadstool toxin $\alpha$-amanitin to inhibit two RNA polymerase activities, which they called A and B, isolated from calf thymus. Eukaryotic RNA polymerase activity was known to be inhibited by the toxin, in contrast to RNA polymerase from Escherichia coli. Chambon and colleagues showed that polymerase A activity was insensitive to $\alpha$-amanitin, whereas polymerase B activity was inhibited. In addition, their study showed that $\alpha$-amanitin affected the elongation

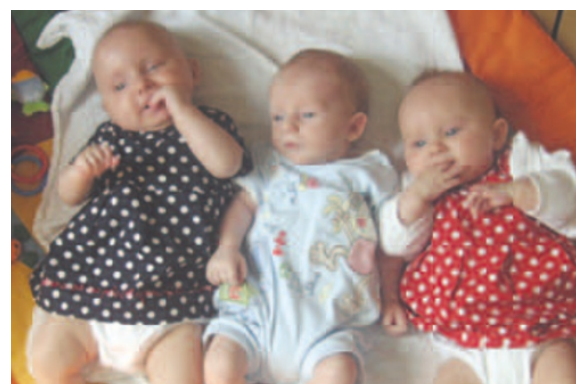

stage of RNA transcription. This work supported previous studies indicating that the bacterial RNA polymerase differed from eukaryotic RNA polymerases, and allowed the researchers to speculate that there were structural differences between polymerases A (I) and B (II).

Shortly thereafter, several laboratories showed that distinct forms of RNA polymerases could be distinguished based on their sensitivities to $\alpha$-amanitin — with polymerase I being insensitive, polymerase II being inhibited and polymerase III being only moderately affected. By correlating the $\alpha$-amanitin sensitivities of the isolated enzymes with the concentrations of $\alpha$-amanitin required to inhibit synthesis of different classes of RNA in cells, these studies supported the idea that RNA polymerases I, II and III, respectively, are responsible for the synthesis of the major rRNAs, mRNA and small RNAs (including 5S RNA, tRNA and a subset of the small-nuclear RNAs). Despite these differences in gene targets, the later discovery that the TATA-binding protein was a transcription factor used by all three polymerases enforced the evolutionary relatedness of these enzymes.

Michelle Montoya, Associate Editor Nature Structural \& Molecular Biology

\section{References and links}

ORIGINAL RESEARCH PAPERS Roeder, R. G. \& Rutter, W. J. Multiple forms of DNA-dependent RNA polymerase in eukaryotic organisms. Nature 224, 234-237 (1969)| Kedinger, C.,

Gniazdowski, M., Mandel, J. L. Jr, Gissinger, F. \& Chambon, P. $\alpha$-amanitin: a specific inhibitor of one of two DNA-dependent RNA polymerase activities from calf thymus. Biochem. Biophys. Res. Commun. 38, 165-171 (1970)

FURTHER READING Lobo, S. M., Lister, J., Sullivan, M. L. \& Hernandez, N. The cloned RNA polymerase II transcription factor IID selects RNA polymerase III to transcribe the human U6 gene in vitro. Genes Dev. 5, 1477-1489 (1991) | Margottin, F. et al. Participation of the TATA factor in transcription of the yeast U6 gene by RNA polymerase C. Science 251, 424-426 (1991) Simmen, K. A. et al. TFIID is required for in vitro transcription of the human U6 gene by RNA polymerase III. EMBO J. 10, 1853-1862 (1991) | Cormack, B. P. \& Struhl, K. The TATAbinding protein is required for transcription by all three nuclear RNA polymerases in yeast cells. Cell 69, 685-696 (1992) | Schultz, M. C., Reeder, R. H. \& Hahn, S. Variants of the TATA-binding protein can distinguish subsets of RNA polymerase I, II, and III promoters. Cell 69, 697-702 (1992) 